\title{
Evaluation of lightning induced voltage due to the effect of design parameters on medium voltage distribution line
}

\begin{abstract}
This paper investigates the effect of design parameters on the induced voltages on a distribution power line. This investigation is based on perfect ground conductivity, single stroke lightning and lightning without branches. The design of the parameters includes, $\mathrm{d}$, the striking distance of the lightning, $h$, the height of the conductor, and $r$, the diameter of the conductor, all of which are elements that produce the variations in the induced voltage on a distribution power line with respect to a vertical or an inclined lightning channel. Thus, the outcome of this investigation can act as a guide for utility companies or other power engineers in order to plan an appropriate protection scheme for a distribution power line.
\end{abstract}

Keyword: Lightning; Induced overvoltage; Striking distance; Vertical lightning channel; Inclined lightning channel 